No. 620

December 2019

The Concept of Prehandling as Direct

Preconditioning for Poisson-like Problems

D. Ruda, S. Turek, P. Zajac, D. Ribbrock

ISSN: 2190-1767 


\title{
The Concept of Prehandling as Direct Preconditioning for Poisson-like Problems
}

\author{
Dustin Ruda, Stefan Turek, Peter Zajac and Dirk Ribbrock
}

\begin{abstract}
To benefit from current trends in HPC hardware, such as increasing availability of low precision hardware, we present the concept of prehandling as a direct way of preconditioning and the hierarchical finite element method which is exceptionally well-suited to apply prehandling to Poisson-like problems, at least in 1D and 2D. Such problems are known to cause ill-conditioned stiffness matrices and therefore high computational errors due to round-off. We show by means of numerical results that by prehandling via the hierarchical finite element method the condition number can be significantly reduced (while advantageous properties are preserved) which enables us to obtain sufficiently accurate solutions to Poisson-like problems even if lower computing precision (i.e. single or half precision format) is used.
\end{abstract}

\section{Motivation}

When PDEs are solved numerically by the finite element method, the resulting error $u-\tilde{u}_{h}$ can be subdivided into two different types of errors by means of the identity $u-\tilde{u}_{h}=\left(u-u_{h}\right)+\left(u_{h}-\tilde{u}_{h}\right)$, whereby $u, u_{h}$ and $\tilde{u}_{h}$ denote the exact solution, the exact solution to the discrete problem and the actual numerical solution respectively. On the one hand, one obtains the discretization error $u-u_{h}$ depending on the smoothness of the exact solution $u$ and the choice of the finite element space. If (bi)linear shape functions are used ( $\mathrm{P} 1$ or $\mathrm{Q} 1)$, the discretization error satisfies $\left\|u-u_{h}\right\|=O\left(h^{2}\right)$ with respect to the $L^{2}$-norm. On the other hand, roundoff errors cause the computational error $u_{h}-\tilde{u}_{h}$ depending on the data error, that is at least equal to the machine accuracy (TOL), amplified by the condition number of the stiffness matrix $\kappa(A)$. To be more precise, it follows from perturbation theory of

D. Ruda $\bullet \mathrm{S}$. Turek $\bullet$ P. Zajac $\bullet$ D. Ribbrock

TU Dortmund, Chair of Applied Mathematics and Numerics (LS3), 44227 Dortmund, Germany

e-mail: dustin.ruda@math.tu-dortmund.de; stefan.turek@math.tu-dortmund.de;

peter.zajac@math.tu-dortmund.de; dirk.ribbrock@math.tu-dortmund.de 
linear systems that we have $\left\|u_{h}-\tilde{u}_{h}\right\| \approx \kappa(A) \cdot$ TOL which holds true sharply. It is known that in the case of Poisson's equation the condition number of the related stiffness matrix satisfies $\kappa(A)=O\left(h^{-2}\right)$.

Consequently, we face a dilemma: The finer the grid the lower the discretization error the higher the computational error. Indeed, if the grid width falls below a certain value, the total error increases because the computational error becomes dominant as shown in Fig. 1. This value is roughly reached at the intersection of the

Fig. $1 L^{2}$-error when solving Poisson's equation with FEM as a function of the refinement level, i.e. $h=2^{\text {-level }}$, in $1 \mathrm{D}$ using single-, double- and quad precision.

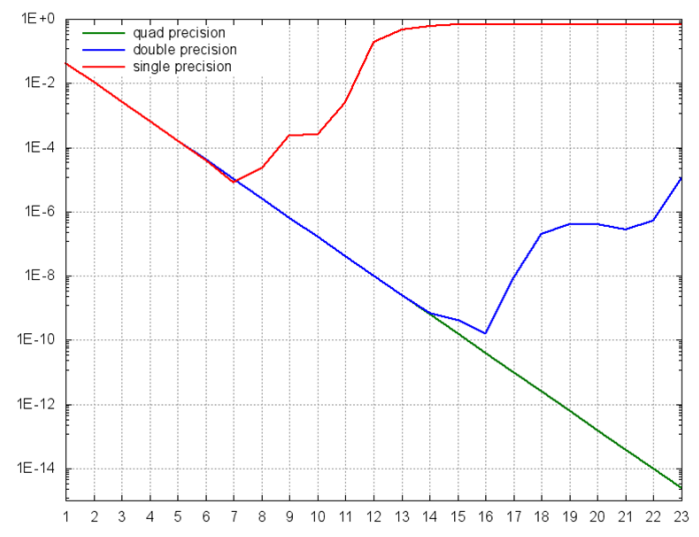

discretization and the computational error at $h \approx \sqrt{\kappa(A) \cdot \text { TOL }}$, yielding $h \approx \sqrt[4]{\mathrm{TOL}}$ if we substitute the condition number by its approximate value $h^{-2}$. Thus, in order to make sensible use of lower, i.e. single or even half precision (accelerator) hardware, it is indispensable to utilise sophisticated methods to decrease the condition number. Our approach, the method of prehandling, is presented in the following sections.

\section{The Concept of Prehandling}

By 'prehandling' we denote a method of directly manipulating linear systems of equations inspired by but different from conventional preconditioning. The central idea consists in transforming the original linear system, given as $A x=b$, into an equivalent form $\tilde{A} \tilde{x}=\tilde{b}, B \tilde{x}=x$ with more advantageous properties. The difference between preconditioning and prehandling can be easily shown by the example of the Richardson iteration. The preconditioned version is

$$
x^{I+1}=x^{I}-C^{-1}\left(A x^{I}-b\right),
$$

whereas by applying prehandling one obtains

$$
x^{I+1}=x^{I}-\left(C^{-1} A x^{I}-C^{-1} b\right)=x^{I}-\left(\tilde{A} x^{I}-\tilde{b}\right) .
$$


Thus, prehandling can be seen as an explicit form of preconditioning. Assuming exact arithmetic and using the exact application of $C^{-1}$, both methods yield the same iteration vectors $x^{I}$ for all $I$. However, the methods (1) and (2) can yield significantly different results when finite precision arithmetic is applied, especially if the matrix $A$ is ill-conditioned.

As mentioned in Section 1, finite element stiffness matrices arising from Poisson's equation are highly ill-conditioned. Yet, an advantageous property is their sparse structure. Via prehandling we intend to reduce the condition number while preserving the sparsity of the matrix. A lower condition number is desirable because it enables us to obtain relevant solutions using fast lower precision hardware and reduces the number of iterations when solving the linear system. To sum up, the three central requirements for the prehandled system are:

1. Strong decrease of the condition number, $\kappa(\tilde{A}) \ll \kappa(A)$.

2. The matrix $\tilde{A}$ is only moderately less sparse than $A$.

3. There is an efficient transformation (in $O(n \log n)$ operations for $n$ unknowns) to $\tilde{A}, \tilde{b}$ and the solution to the original system $x$ (via $x=B \tilde{x}$ ).

A common method is approximating the inverse of $A$ by a matrix $C \approx A^{-1}$ and computing $\tilde{A}=C A$. For this purpose, one can use e.g. matrix splitting $\left(C=D^{-1},(D+L)^{-1}\right)$, incomplete LU-Decomposition (ILU) or the Sparse Approximate Inverse (SPAI) but the requirements 1 . and 2. are not satisfied by any of these methods.

A promising technique to meet the demands (at least in 1D and 2D) is the hierarchical finite element method presented in the following section.

\section{The hierarchical Finite Element Method}

The hierarchical finite element method (also referred to as hierarchical basis multigrid method, abbr.: HFEM) has been known since the 1980s and was developed and analysed by $\mathrm{H}$. Yserentant et al. in [1], amongst others. The main idea and aspects of the realisation as well as the properties that make this method a proper choice for prehandling are shortly outlined.

\subsection{Idea and Realisation}

In order to apply this method, a nested sequence of refinements of an initial triangulation is required. The general idea is the usage of a hierarchical instead of a nodal basis. This means that basis functions of coarser grids are reused in the course of the refinement. Fig. 2 shows nodal compared to hierarchical bases in one dimension. This concept can be straightforwardly applied to higher dimensions, too. 
It seems more complicated to assemble the stiffness matrix with respect to a hierarchical basis since many basis functions have a greater support, but it is in fact not necessary to compute the matrix itself in the first place if the stiffness matrix with respect to a nodal basis is known. Instead, we can transform the nodal basis representation to a hierarchical basis representation via a matrix $S$. It is computed as the product $S=S_{j} S_{j-1} \cdots S_{1}$, whereby each factor is associated with one step of refinement. More precisely, multiplying by $S_{k}$ yields the values of level $k-1$ basis functions at the new nodes of level $k$ if the values on the coarser grid are known. In other words, the matrix $S_{k}$ corresponds to the prolongation regarding multigrid methods. The matrices $S_{k}$ are concretely computed as follows: They are identity matrices with additional entries in the rows that belong to the newly added nodes of level $k$. When using a triangular mesh with linear basis functions (P1) and uniform refinement (subdividing each triangle into four congruent triangles), each newly added node of level $k$ with index $i$ has two neighbouring nodes on level $k-1$, which are denoted by $n_{1}(i), n_{2}(i)$, and the $i$ th row of $S_{k}$ is adjusted according to

$$
S_{k}\left(i, n_{1}(i)\right)=S_{k}\left(i, n_{2}(i)\right)=\frac{1}{2} .
$$

When using a rectangular 2D mesh with bilinear basis functions (Q1), however, uniform refinement generates two different types of new nodes, namely midpoints of edges with two and midpoints of faces with four neighbouring nodes $n_{j}(i)$. In this case one needs to apply

$$
S_{k}\left(i, n_{j}(i)\right)= \begin{cases}\frac{1}{2}, j=1,2, & \text { if } x_{i} \text { is midpoint of edge } \\ \frac{1}{4}, j=1, \ldots, 4, & \text { if } x_{i} \text { is midpoint of face }\end{cases}
$$

to achieve a correct interpolation.

Due to this construction, $S$ is a very sparse block unit lower triangular matrix. If by $\hat{A} \hat{u}=\hat{b}$ we denote the system with respect to the nodal basis, we obtain the representation with respect to the hierarchical basis $A u=b$ by means of the transformation

$$
A=S^{\top} \hat{A} S, b=S^{\top} \hat{b}, \hat{u}=S u .
$$

Fig. 2 Nodal bases (left) and hierarchical bases (right) in the one-dimensional case assuming homogeneous Dirichlet boundary conditions. Note that for the hierarchical bases only the newly added basis functions of the respective meshes are depicted.

Source: [2]

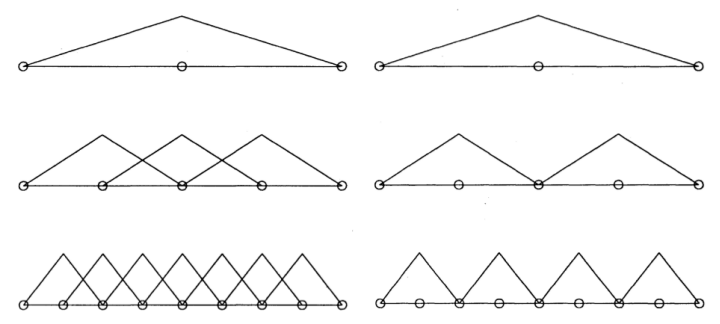




\subsection{Properties}

The remarkable property about the hierarchical finite element method is that the condition number of the emerging matrix is significantly lower in comparison to standard finite elements. It was shown in [1] that the spectral condition number of the matrix with respect to the hierarchical basis satisfies

$$
\kappa(A)=O\left(\left(\log \frac{1}{h}\right)^{2}\right)
$$

in the one- and two-dimensional case which is a strong improvement compared to

$O\left(h^{-2}\right)$. Furthermore, the matrix $A$ is obviously denser than $\hat{A}$, but the number of nonzero entries per row is low enough to be referred to as sparse and due to the sparse structure of the matrix $S$, the transformation can be realised efficiently. Numerical results that show the reduction of the condition number and the sparsity of the transformed matrix (approx. 16 nonzero entries per row - if an orthogonal mesh is used - compared to 9 without prehandling) are given in Section 4, Table 1.

In conclusion, the hierarchical finite element method, if used according to the concept of prehandling, satisfies the required properties, at least in one and two dimensions. In the three-dimensional case, though, the same improvement of the condition number cannot be achieved. Instead, it is shown in [3] that $\kappa(A)=O\left(\frac{1}{h} \log \frac{1}{h}\right)$ respectively $O\left(\frac{1}{h}\right)$ if further prehandling is used.

\subsection{Additional Prehandling via Partial Cholesky Decomposition}

If Poisson's equation with a discontinuous coefficient $\varrho$

$$
-\nabla \cdot(\varrho \nabla u)=f \text { in } \Omega
$$

whereby $\varrho(x, y)=1$ in a subdomain $\Omega_{1} \subset \Omega$ and $\varrho(x, y)=\varrho \gg 1$ in $\Omega \backslash \Omega_{1}$, is considered, the condition number of the stiffness matrix with respect to a nodal basis additionally depends on the ratio $\frac{\max (\varrho)}{\min (\varrho)}$ (denoted by $\Delta \varrho$ ) in the form of $\kappa(\hat{A})=O\left(\Delta \varrho \cdot h^{-2}\right)$. Especially in this case but also in the case of standard Poisson's equation $(\varrho=1)$ there is a powerful way for further prehandling using a partial Cholesky decomposition presented in $[1,2]$.

By $A_{0}$ we denote the part of the matrix $A$ (which is assumed to be represented with respect to a hierarchical basis) that corresponds to the coarse grid and the rest of it by $\tilde{A}$ and compute the following Cholesky decomposition

$$
\left(\begin{array}{cc}
A_{0} & 0 \\
0 & \operatorname{diag}(\tilde{A})
\end{array}\right)=L L^{\top} .
$$


Consequently, we get the additionally prehandled matrix and right hand side as $L^{-1} A L^{-\top}$ respectively $L^{-1} b$. Note that the solution needs to be transformed to the nodal basis representation by multiplication with $S L^{-\mathrm{T}}$.

It shows in our numerical test on an orthogonal 2D mesh (see Section 4, Table 2) that the condition number of $L^{-1} A L^{-\top}$ is now virtually independent of $\Delta \varrho$. The only condition is that the coefficient $\varrho$ is constant within the interior of the elements of the coarse grid.

Since the matrix $A_{0}$ is very small compared to $A$, the computational cost of the Cholesky decomposition (8) is low. On the other hand, the further prehandling only works to the disadvantage of the sparsity, but if the coarse grid is not chosen too fine, this effect is not excessive (approx. 16 - 25 nonzero entries per row if the coarse grid width is greater or equal to $\left.h_{0}=1 / 8\right)$ as one can see in Section 4, Tables 1, 2 .

\section{Numerical Results}

In order to validate the presented methods, they were practically applied to the Poisson-like equation (7) in the unit square $\bar{\Omega}=[0,1]^{2}$ with $f=1$ and

$$
\varrho(x, y)=\left\{\begin{array}{ll}
\varrho, & \text { if }(x, y) \in\left[\frac{1}{4}, \frac{3}{4}\right]^{2} . \\
1, & \text { else }
\end{array} .\right.
$$

Two different widths $h$ of the fine grid and three widths $h_{0}$ of the coarse grid in each case were chosen and the density measured as the average number of nonzero entries per row (NNZ/Row) and the spectral condition number of the respective matrix (cond) as well as the number of CG-iterations (NOI) necessary to reach a relative residual less than $10^{-6}$ were determined for the hierarchical method with and without the additional Cholesky prehandling and the plain finite element method for comparison. Q1 finite elements on a grid consisting of squares were used. Table 1 shows the results in the case $\varrho=1$ (which yields the standard Poisson's equation) and Table 2 in the case $\varrho=10^{6}$.

Table 1 Results for Poisson's equation $(\varrho=1)$.

\begin{tabular}{|c|c|c|c|c|c|c|c|c|c|c|}
\hline \multirow[b]{2}{*}{$h$} & \multirow[b]{2}{*}{$h_{0}$} & \multirow{2}{*}{\multicolumn{2}{|c|}{$\begin{array}{c}\text { HFEM } \\
\text { cond }\end{array}$}} & \multirow[b]{2}{*}{ NOI } & \multicolumn{3}{|c|}{ HFEM + Chol. } & \multirow[b]{2}{*}{$\frac{\text { NNZ }}{\text { Row }}$} & \multirow{2}{*}{$\begin{array}{l}\text { FEM } \\
\text { cond }\end{array}$} & \multirow[b]{2}{*}{ NOI } \\
\hline & & & & & $\frac{\text { NNZ }}{\text { Row }}$ & cond & NOI & & & \\
\hline \multirow{3}{*}{$1 / 64$} & $1 / 4$ & 15,61 & 23,39 & 27 & 16,20 & 20,92 & 23 & \multirow{3}{*}{8,81} & \multirow{3}{*}{829,86} & \multirow{3}{*}{63} \\
\hline & $1 / 8$ & 15,38 & 27,51 & 27 & 25,25 & 15,21 & 20 & & & \\
\hline & $1 / 16$ & 14,51 & 82,96 & 32 & 100,12 & 9,62 & 14 & & & \\
\hline \multirow{3}{*}{$1 / 128$} & $1 / 4$ & 16,68 & 31,57 & 32 & 16,99 & 28,76 & 27 & \multirow{3}{*}{8,91} & \multirow{3}{*}{$3.319,93$} & \multirow{3}{*}{127} \\
\hline & $1 / 8$ & 16,56 & 33,30 & 33 & 21,79 & 22,08 & 24 & & & \\
\hline & $1 / 16$ & 16,11 & 92,21 & 37 & 65,40 & 15,44 & 19 & & & \\
\hline
\end{tabular}


Table 2 Results for the Poisson-like equation $\left(\varrho=10^{6}\right)$.

\begin{tabular}{cc|ccc|ccc|ccc}
\hline & & \multicolumn{4}{c|}{ HFEM } & \multicolumn{3}{|c|}{ HFEM + Chol. } & \multicolumn{3}{c}{ FEM } & \\
$h$ & $h_{0}$ & NNZ & cond & NOI & $\frac{\text { NNZ }}{\text { Row }}$ & cond & NOI & $\frac{\text { NNZ }}{\text { Row }}$ & cond & NOI \\
\hline \multirow{3}{*}{$1 / 64$} & $1 / 4$ & 16,03 & $1,57 \cdot 10^{7}$ & 623 & 16,20 & 22,48 & 21 & & & \\
& $1 / 8$ & 15,61 & $2,01 \cdot 10^{7}$ & 693 & 25,26 & 15,52 & 21 & 8,81 & $7,05 \cdot 10^{8}$ & 2.331 \\
& $1 / 16$ & 14,61 & $6,86 \cdot 10^{7}$ & 783 & 100,66 & 9,67 & 17 & & & \\
\hline \multirow{3}{*}{$1 / 128$} & $1 / 4$ & 17,31 & $2,23 \cdot 10^{7}$ & 892 & 16,99 & 30,64 & 25 & & & \\
& $1 / 8$ & 16,98 & $2,44 \cdot 10^{7}$ & 1.042 & 21,77 & 22,48 & 25 & 8,91 & $2,82 \cdot 10^{9}$ & 8.431 \\
& $1 / 16$ & 16,34 & $7,61 \cdot 10^{7}$ & 1.110 & 65,53 & 15,52 & 21 & & & \\
\hline
\end{tabular}

One can observe a vast decrease of the condition number and thus the number of iterations when the matrix is transformed to a hierarchical basis representation when Poisson's equation is considered. The method of further prehandling by a partial Cholesky decomposition turns out to be very robust with respect to $\varrho$ in sharp contrast the other listed methods as Table 2 shows.

Furthermore, it was tested if the application of the hierarchical method actually enables us to lower the error when using single or even half precision floatingpoint format. The course of the $L^{2}$-error depicted in the figures 3 (1D) and 4 (2D) show that by prehandling via the hierarchical method one achieves more accurate approximations in single and double precision in the 1D case and in half and single precision in the 2D case compared to the finite element method. For more detailed and further numerical results, such as P1 finite elements and rectangular domains (where the hierarchical method has basically identical effects), we refer to [4].

Fig. $3 L^{2}$-error when solving Poisson's equation with prehandling by HFEM as a function of the refinement level, i.e. $h=2^{- \text {level }}$, in 1D using single, double and quad precision.

Compare to Fig. 1.

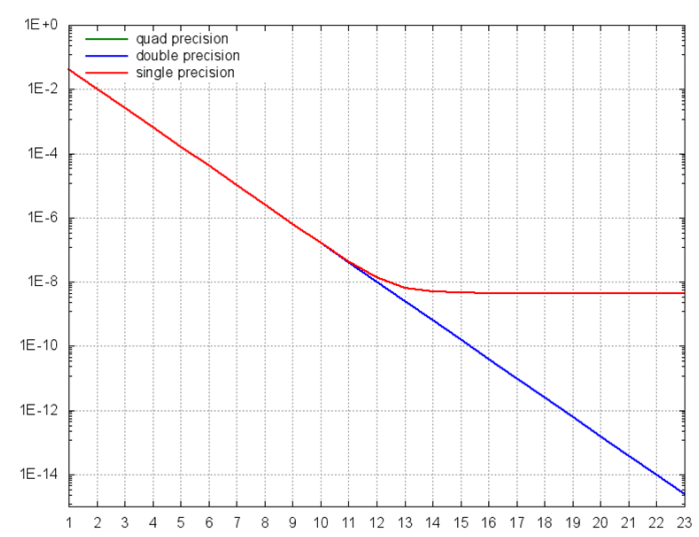



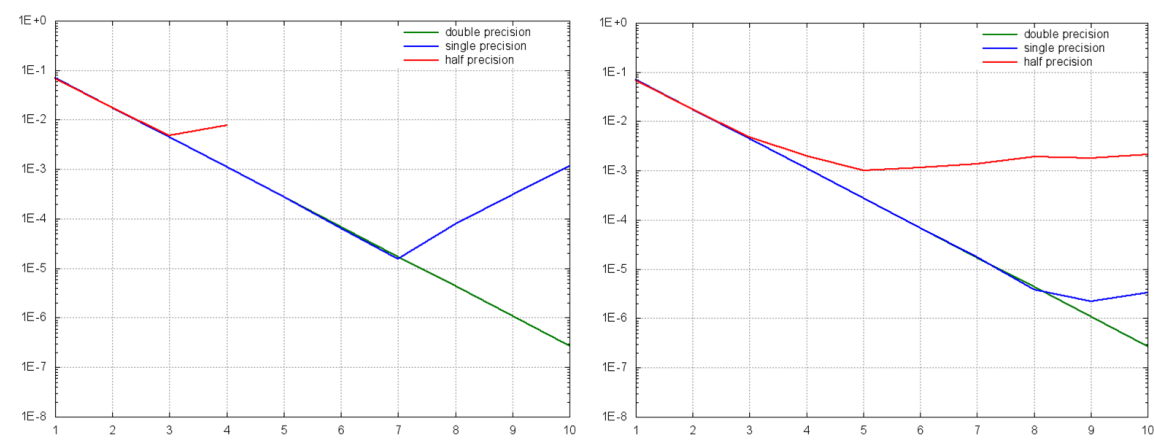

Fig. $4 L^{2}$-error with FEM (left) and prehandling by HFEM (right) as a function of the refinement level in $2 \mathrm{D}$ using half, single, and double precision.

\section{Summary and Conclusion}

By the concept of prehandling together with the hierarchical finite element method the requirements of a lower condition number, preservation of sparsity and an efficient transformation are successfully met in the case of Poisson's equation in 1D and 2D as numerical results confirm. This allows us to use low precision hardware without losing too much accuracy. In the context of technical applications where an error of $1 \%$ is often satisfactory, even the usage of half precision might be appropriate. Additionally on the plus side, the expense of the iterative solution to the linear system is reduced if the hierarchical method is applied. By implementing further prehandling via a partial Cholesky decomposition, we can also deal with the case of the Poisson-like equation with a jumping coefficient. A central task for future research is to extend this method to apply prehandling in $3 \mathrm{D}$, too.

\section{Acknowledgements}

The results presented in Fig. 1, 3, 4 have been created using the FEM software package FEAT3 ${ }^{1}$.

\section{References}

1. Yserentant, H.: On the Multi-Level Splitting of Finite Element Spaces. In: Numer. Math. 49, pp. 379-412. Springer (1986)

2. Deuflhard, P., Leinen, P., Yserentant, H.: Concepts of an Adaptive Hierarchical Finite Element Code. In: IMPACT of Computing in Science and Engineering 1, pp. 3-35. Academic Press, Inc. (1989)

3. Ong, M. E. G.: Hierarchical Basis Preconditioners in Three Dimensions. In: SIAM J. Sci. Comput. 18 (1997), pp. 479-498.

4. Ruda, D.: Numerische Studien zur "Vorbehandlung" (prehandling) von Poisson-artigen Problemen durch die hierarchische Finite-Elemente-Methode. Master thesis, TU Dortmund, to appear 2020

${ }^{1}$ see http://www.featflow.de/en/software/feat3.html 